\title{
Professeur Jean-Patrick Schaal
}

\section{Riethmuller $\cdot$ R. Maillet}

C Springer-Verlag France 2011

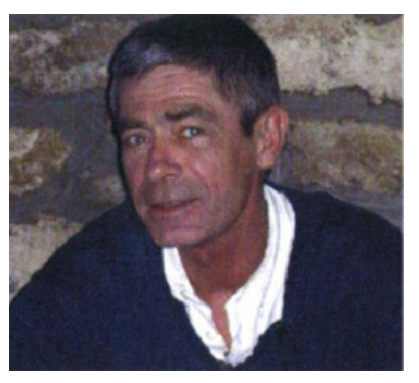

Le 21 décembre 2010, juste avant Noël, Jean-Patrick Schaal nous a quittés après quelques mois d'une terrible maladie. La très vive et unanime émotion qu'a suscitée sa disparition prouve le caractère exceptionnel du personnage.

L'obstétrique française a perdu non seulement un incontestable leader, mais surtout un de ses principaux piliers. Son infaillible implication pour la discipline va laisser un vide immense que nous nous efforcerons de combler.

Il a été Pr de gynécologie-obstétrique, puis chef de service au CHU de Besançon de 2000 à 2004 avant de prendre la responsabilité du service universitaire de gynécologieobstétrique de Grenoble, sa ville de faculté.

La somme de travail qu'il a fournie sur ces deux sites et les avancées qu'il y a générées sont si considérables et si nombreuses qu'il n'est pas possible de toutes les citer.

Il a été un fervent acteur de la diffusion et de l'enseignement de la mécanique et des techniques obstétricales (MTO) pour le bien des mères et des nouveau-nés. Il a œuvré durant toute sa carrière pour l'enseignement de l'art des accouchements ; beaucoup aujourd'hui se sentent ses élèves, et tous savent ce qu'ils lui doivent.

Il est l'auteur de plusieurs ouvrages reconnus comme étant des références et qui vont encore longtemps permettre aux plus jeunes de se passionner pour cette incroyable aventure humaine qu'est la parturition.

Lorsqu'en 1990, il a créé contre vents et marées le diplôme universitaire de MTO, personne n'a cru à ce projet prétextant que la connaissance de l'obstétrique était un prérequis à l'entrée dans la filière du DES de gynécologie-obstétrique. En
2011, ce sont cinq CHU de France qui dispenseront cet enseignement. La liste d'attente des étudiants demandeurs de cette formation, qui aujourd'hui est un DIU, est très longue : quel succès ! Ce succès a même dépassé les frontières de notre hexagone puisqu'une version internationale de ce diplôme sera enseignée dès cette année.

Il a redonné ses lettres de noblesse à l'évaluation du bien-être fotal au cours du travail et a transmis le sens de la rigueur et du pragmatisme dans la lecture de l'enregistrement du rythme cardiaque fotal et du tocogramme, tout comme cela se fait pour la lecture de l'électrocardiogramme.

Inventeur d'une nouvelle ventouse obstétricale, sa passion pour le monde de l'informatique qui porte le sigle de la « pomme » l'a fait nommer « iCup ${ }^{\circledR} »$. Elle fait actuellement l'objet d'une évaluation dans le cadre d'un protocole hospitalier de recherche clinique à l'échelon national. La ventouse obstétricale n'avait pas de meilleur expert en France et ses très nombreuses présentations et publications sur le sujet ont abouti en 2007 à cette réalité : la ventouse obstétricale, outil marginal dans les salles d'accouchement avant 1990, est devenue l'instrument d'extraction le plus usité dans les centres académiques de notre pays.

Sur le plan humain, ceux qui comme nous ont eu la chance de bien connaître cet homme de conviction et qui ont eu l'immense joie d'être son ami savent qu'ils pouvaient toujours compter sur lui, sur sa fidélité, son déterminisme et son aide affectueuse.

Jean-Patrick Schaal est en fait inscrit dans une longue tradition obstétricale qui a ses racines à Nancy d'où est issu notre patron, le Pr Colette, qui nous a enseigné l'obstétrique. Mais, c'est Jean-Patrick qui est à l'origine de « l'École de Besançon » et nous sommes résolus à faire perdurer cette école pour que l'obstétricien Jean-Patrick soit toujours vivant dans nos mémoires.

D. Riethmuller $(\bowtie) \cdot$ R. Maillet $(\bowtie)$

Service de gynécologie obstétrique,

CHU Saint-Jacques,

F-25030 Besançon cedex, France

e-mail : didier.riethmuller@univ-fcomte.fr,

robert.maillet@univ-fcomte.fr 